JSS Focus Issue ON THermoelectric Materials \& Devices

\title{
Review-Micro and Nano-Engineering Enabled New Generation of Thermoelectric Generator Devices and Applications
}

\author{
Jhonathan P. Rojas, ${ }^{\text {a,z }}$ Devendra Singh, ${ }^{\mathrm{b}}$ Salman B. Inayat, ${ }^{\mathrm{c}}$ Galo A. Torres Sevilla, ${ }^{\mathrm{b}}$ \\ Hossain M. Fahad, ${ }^{b}$ and Muhammad M. Hussain ${ }^{b, z}$ \\ ${ }^{a}$ Electrical Engineering Department, King Fahd University of Petroleum and Mineral, Dhahran 31261, Saudi Arabia \\ ${ }^{b}$ Integrated Nanotechnology Lab and Integrated Disruptive Electronic Applications (IDEA) Lab, King Abdullah \\ University of Science and Technology, Thuwal 23955, Saudi Arabia \\ ${ }^{c}$ TSI Semiconductors, Roseville, California 95747, USA
}

\begin{abstract}
As we are advancing our world to smart living, a critical challenge is increasingly pressing - increased energy demand. While we need mega power supplies for running data centers and other emerging applications, we also need instant small-scale power supply for trillions of electronics that we are using and will use in the age of Internet of Things (IoT) and Internet of Everything (IoE). Such power supplies must meet some parallel demands: sufficient energy supply in reliable, safe and affordable manner. In that regard, thermoelectric generators emerge as important renewable energy source with great potential to take advantage of the widely-abundant and normally-wasted thermal energy. Thanks to the advancements of nano-engineered materials, thermoelectric generators' (TEG) performance and feasibility are gradually improving. However, still innovative engineering solutions are scarce to sufficiently take the TEG performance and functionalities beyond the status-quo. Opportunities exist to integrate them with emerging fields and technologies such as wearable electronics, bio-integrated systems, cybernetics and others. This review will mainly focus on unorthodox but effective engineering solutions to notch up the overall performance of TEGs and broadening their application base. First, nanotechnology's influence in TEGs' development will be introduced, followed by a discussion on how the introduction of mechanically reconfigurable devices can shape up the emerging spectrum of novel TEG technologies.

(C) The Author(s) 2017. Published by ECS. This is an open access article distributed under the terms of the Creative Commons Attribution Non-Commercial No Derivatives 4.0 License (CC BY-NC-ND, http://creativecommons.org/licenses/by-nc-nd/4.0/), which permits non-commercial reuse, distribution, and reproduction in any medium, provided the original work is not changed in any way and is properly cited. For permission for commercial reuse, please email: oa@electrochem.org. [DOI: 10.1149/2.0081703jss] All rights reserved.

(cc) BY-NC-ND
\end{abstract}

Manuscript submitted September 21, 2016; revised manuscript received December 14, 2016. Published January 12, 2017. This paper is part of the JSS Focus Issue on Thermoelectric Materials \& Devices: Phonon Engineering, Advanced Materials and Thermal Transport.

The technology-driven age we are currently in, have brought great advantages to establish a more comfortable and smarter living and it is leading the way for an even faster-growing development in all areas of science and engineering, but at the same time it brings an incredibly fast growing demand for energy. Current and future energy consumption mandate the need for alternative energy sources, with reduced environmental impact. Readily available solar and wind energies have lead the way as alternative energy sources to environmentally challenging fossil fuels. ${ }^{1}$ Moreover, thanks to more recent developments in thermoelectric (TE) materials and devices, the possibility of making a better use of the widely abundant and normally wasted thermal energy, has becoming a popular and feasible alternative. ${ }^{2}$ More importantly, thermal waste has become a very important and environmentallyfriendly source of otherwise wasted energy. ${ }^{3,4}$ There has been already an important set of studies covering the mechanism involved during the conversion from thermal to electric energy. ${ }^{5-8}$ Such studies have been the starting point to develop and optimize novel TE materials with the resourceful aid of uprising nanotechnologies. ${ }^{9,10}$ In this review, we will first shortly introduce some important basic concepts and relations about thermoelectrics, as well as some of the current efforts to use nanotechnologies and nano-materials to improve performance and viability. Next, we will focus on identifying innovative devices, novel engineering approaches, and applications with great potential of contributing to solve contemporary real-world challenges. In this regard, we will delve into a very important feature that nowadays has become a game changer in terms of technology's functionality and practicality; the ability of electronic systems to become mechanically adaptable and reconfigurable. This implies that the new era of electronic devices is arriving with the additional mechanical complexity of displaying flexibility and stretchability for full integration with irregular, curvilinear, biological and mobile systems. ${ }^{1-13}$ At the same time, along with this mechanical and architectural shift in electron-

${ }^{\text {z} E-m a i l: ~ M u h a m m a d M u s t a f a . H u s s a i n @ k a u s t . e d u . s a ; ~ j p r o j a s @ k f u p m . e d u . s a ~}$ ics, it is imperative to ensure that complementary technologies, which are also part of a whole electronic system (such as sensors, antennas and energy harvesting and storage devices), also follow the same shift toward mechanical reconfigurability, thus enabling the rise and evolution of technologies for a smarter and sustainable future.

\section{Principles of Thermoelectrics}

The conversion from thermal to electric energy has been well known for a long time. ${ }^{14}$ More recently, thermoelectric generators (TEGs) have demonstrated numerous advantages such as the use of solid-state-based devices, lack of moving parts, which ensures their long-term lifetime, diversity and flexibility of fabrication, as well as the ability of certain TE materials to be scaled down to nanoscale dimensions for improved performance. ${ }^{15}$ However, it remains a challenge to successfully integrate them with portable devices due to their low figure of merit (ZT). To understand the efficiency of any TE material, ZT can easily be represented as the tendency of any material to efficiently convert a thermal gradient into electrical power. It can be defined as following:

$$
Z T=\frac{S^{2} \sigma T}{\kappa}
$$

Where $S\left(\mathrm{~V} \mathrm{~K}^{-1}\right)$ is the Seebeck coefficient, $\sigma\left(\mathrm{S} \mathrm{m}^{-1}\right)$ is electrical conductivity, $T(\mathrm{~K})$ is temperature and $\kappa\left(\mathrm{W} \mathrm{K}^{-1} \mathrm{~m}^{-1}\right)$ is the thermal conductivity. ${ }^{16}$ The maximum electro-thermal energy conversion efficiency and maximum cooling temperature can be determined by $Z$ value. Because $Z$ has a unit of inverse temperature, the nondimensional figure of merit $Z T$ is often used.

For many decades, the progress in the development of disruptively highly efficient TE materials has been excruciatingly slow. Materials with good electrical and low thermal conductivity are strongly needed for best output efficiencies. As these needed behaviors are affected by electrical properties and their complex interactions, it is very difficult 
to optimize this challenging criteria. Additionally, for semiconductors, it is desirable to have a base material that can be both p- and n-type doped, so that the same material system can be used on both sides of the junctions.

Several metrics have been defined to evaluate the performance of a TEG. For example, the maximum power density of a TEG can be expressed by the following equation:

$$
P_{\max }=\left(\frac{T_{h}-T_{c}}{T_{h}}\right) Q \frac{\sqrt{Z T+1}-1}{\sqrt{Z T+1}-1+\frac{T_{c}}{T_{h}}}
$$

Where $Q$ is the rate of heat transfer through the generator, $T_{\mathrm{h}}$ is the temperature at the hot junction, and $T_{\mathrm{c}}$ is the temperature at the surface being cooled. ${ }^{15}$

On the other hand, there can be found several TEG's efficiency definitions. A common one is given by the following equation: ${ }^{17}$

$$
\eta^{\prime}=\frac{\text { Energy supplied to the load }}{\text { Heat energy absorbed at hot junction }}
$$

Where the energy supplied to the load is the output power of the TEG and the heat energy absorbed at the hot junction is the input power of the TEG. If we now take $Z T_{a v}$ as the average value of both p- and n-type legs, over the temperature dependent ZT curve between $T_{h}$ and $T_{c}$, the power-generation-efficiency can be define as: $:^{18,19}$

$$
\eta_{p}=\frac{T_{h}-T_{c}}{T_{h}}\left[\frac{\sqrt{1+Z T_{a v e}}-1}{\sqrt{1+Z T_{a v e}}+\frac{T_{c}}{T_{h}}}\right]
$$

Where, $Z T_{\text {ave }}=\frac{1}{T_{h}-T_{c}} \int_{T_{c}}^{T_{h}} Z T d T$

Similarly, the thermoelectric cooling efficiency in terms of $\mathrm{ZT}_{\mathrm{av}}$ can be given by: ${ }^{18,19}$

$$
\eta_{c}=\frac{T_{h}}{T_{h}-T_{c}}\left[\frac{\sqrt{1+Z T_{a v e}}-\frac{T_{h}}{T_{c}}}{\sqrt{1+Z T_{\text {ave }}}+1}\right]
$$

In semiconductors, the thermal conductivity consists of contributions from electrons and phonons, where the majority contribution comes from phonons. Thus, reducing phonon thermal conductivity is of great interest. Alloying is one of the most practiced approaches through which this can be achieved without causing degradation in the electrical conductivity. Moreover, among various existing materials, insulators have poor electrical conductivity $(\sigma)$ and metals have relatively low Seebeck coefficients. ${ }^{20}$ In addition, the thermal conductivity $(\kappa)$ of a metal, which is dominated by electrons, is proportional to the electrical conductivity, as dictated by the Wiedemann-Franz law. It is thus difficult to attain a high $Z T$ in conventional metals. On the other hand, highly doped semiconductors can become one of the best thermoelectric materials because heat is carried predominantly by the lattice. In this sense, the thermal conductivity ( $\kappa)$ is largely decoupled from the power factor $\left(S^{2} \sigma\right)$.

To understand the current requirement in terms of efficiency tradeoff, it is reported that module design plays a key factor in determining the cost of waste-heat-recovery thermoelectric systems. Bringing down the material cost from $\$ 1-2$ per watt to $\$ 0.05-0.1$ per watt is also feasible without improving $Z T$ if thin-film modules with hundredsof-micrometers-thick elements with low fractional area coverage (5-10\%) and small parasitic resistance (a contact resistance in the low $10^{-6} \Omega \mathrm{cm}$ ) can be developed. ${ }^{21}$ On the other hand, high figures of merit $(Z T>3.0)$ would be needed in order to replace, for example, conventional chlorofluorocarbon based coolers by thermoelectric coolers. However increasing $Z T$ of bulk semiconductors beyond 1 has remained a challenge. ${ }^{22,17}$ In consequence, novel approaches have been proposed as will be discussed in the following section.

\section{Thermoelectric Device: From Bulk to Nano}

Various thermoelectric materials have been extensively explored and studied including semiconductors to ceramics, different materials system from single crystal to polycrystalline to composites, and

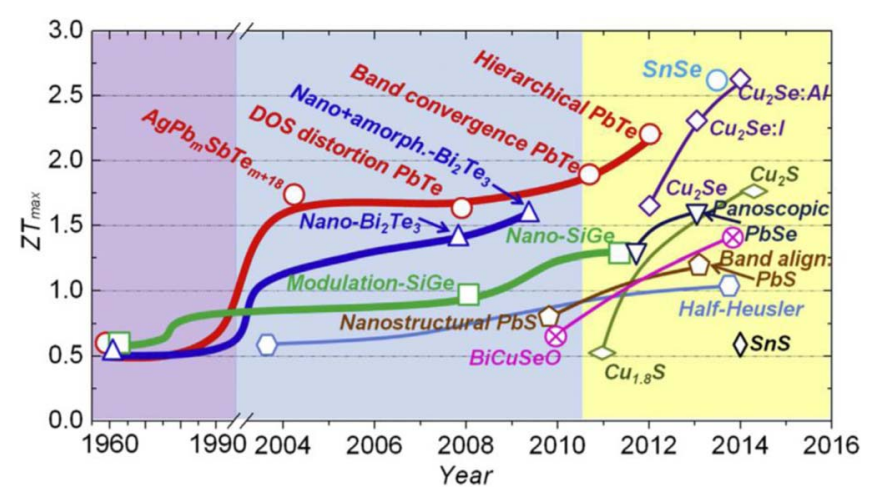

Figure 1. ZT values of several thermoelectric materials as a function of year. ${ }^{30}$

different sizes from bulk, thin-film and one-dimensional structures to cluster. $^{23,24}$ In recent years, many studies on improving the figure of merit $(Z T)$ are moving toward the use of nanostructured materials. ${ }^{15}$ Interestingly, although there have been several demonstrations of $Z T>$ 1 in the past decade, no material has yet achieved the target goal of $Z T$ $\geq 3$ (Figure 1). In fact, the material systems that have achieved $Z T>$ 1 till date, have all been based on systems using nanostructures. ${ }^{25}$ For instance, $\mathrm{Li}$ et al. has demonstrated a microscopic picture connecting the electronic structure and phonon anharmonicity in a single crystal SnSe system. This offered new insights on how electron-phonon and phonon-phonon interactions may lead to the realization of ultralow thermal conductivity. ${ }^{26}$ Nanostructured materials such as alloyed nanosystem, ${ }^{24}$ nanowires, ${ }^{27-32}$ superlattices, ${ }^{33}$ quantum wells, ${ }^{34}$ nanomesh, ${ }^{35}$ nanoribbons ${ }^{36}$ are generally used in the manufacturing of new TE based systems. In fact, thermal conductivity can be significantly reduced owing to nanostructured materials. ${ }^{37}$

Recently, the interest in thermoelectric devices has gained momentum with the advances in nanostructural engineering, which led to overall efforts to demonstrate high-efficiency materials. At the same time, complex bulk materials (such as skutterudites, clathrates, and Zintl phases) have been explored, and it was found that highefficiencies could be obtained. ${ }^{9}$ These complex high-efficiency materials that manage to decouple the conflicting properties have led to many challenges in the field: a wide array of new approaches, from complexity within the unit cell to nano-structured bulk and materials combined in thin-film multi-layer structures, have all led to better and better solutions. ${ }^{9}$

Several approaches have been extensively studied to enhance the thermoelectric performance such as enhancement of Seebeck coefficient through modifications of electronic band structures and band convergence, ${ }^{38-40}$ or reduction of lattice thermal conductivity via allscale hierarchical rendering ${ }^{41}$ and nanostructuring. ${ }^{42}$ It has also been shown that in some cases the enhancement of ZT can be influenced many times more by the lattice thermal conductivity than that of the electric conductivity. ${ }^{43}$ So far, several promising TE materials with intrinsically low thermal conductivities have been reported. Possible explanations to such low values are their liquid like transports, high anharmonicity of chemical bonds, complex crystal structures or large molecular weights. ${ }^{30}$ Additionally, it has been observed that doping of nanowires can be used to alter the scattering of phonons by modifying the surface roughness and size confinement, achieving the reduction of thermal conductivity while keeping the electrical conductivity unaffected, thus highly enhancing the overall ZT value. This technological approach could be encouraging and is compatible with the semiconductor industry. ${ }^{44}$

Concerning polymeric-based TE materials, attempts have been reported to optimize the power factors of conducting polymer-based nanowires. ${ }^{45}$ On the other hand, CNTs and graphene have also been explored to enhance the TE performance of conducting polymers due to their extremely high carrier mobility and unique electronic structures. ${ }^{46,47}$ In this manner, nanocomposites have been used to channel the loss in effective efficiency by optimizing thermal conductivity. 
Moreover, further improvement in power factors of conducting polymers has been shown by addition of inorganic semiconductors. ${ }^{48,49}$

Finally, nanostructured binary metal sulfides have been recently identified as TE materials with great potential due to their great abundance and lower cost and toxicity than traditional TE materials. ${ }^{50}$

In summary, several different techniques and materials have been explored and are in constant evolution to improve efficiency and drive the transition toward a more stablished commercial stage. Nevertheless, it is also very important to identify appropriate niches and applications, as well as ingenious designs and out-of-the-box engineering approaches, to bridge the current performance and affordability gap. Therefore, in the remainder of this review, we will now focus on practical implementations of TEG-based technologies with promising applications in diverse fields, such wearable systems, mass-scale thermoelectric generation and regenerative computing.

\section{Mechanically Adaptable Thermoelectric Generators and Applications}

Thermoelectric generators represent a simple and affordable solution for energy harvesting in applications such as wearable and mobile electronics. However, the use of rigid inorganic semiconductors and substrates makes them unsuitable for applications where mechanical flexibility is of vital importance. For this reason, over the last few years, many different approaches have been made to combine the high efficiency of inorganic thermoelectric materials with the mechanical flexibility and affordability provided by organic materials. Several excellent reviews and reports have already explored the distinct advancements of organic and inorganic materials for flexible TEG applications. ${ }^{51-55}$ In fact, research on naturally-flexible, polymerbased materials have been focusing on improving their TE properties, whereas inorganic-based approaches have been relying on wellknown techniques for their implementation, such as molding ${ }^{56}$ and lithographic patterning. ${ }^{57,58}$ On the other hand, a different approach consist of the beneficial integration and mixing between organics and inorganics to form composite materials with increased functionality and performance; the ideal is to combine the best of each material. For instance, organic conductive polymers, with inherent low thermal conductivity but overall low figures of merit, can display flexibility as well as simple synthesis and processability at a lower cost, whereas inorganic semiconductors with great thermoelectric properties are usually scarce, therefore more expensive, and can be even toxic. By preparing polymer-inorganic TE composites, there is a great potential to reduce the production cost while maintaining a good performance as well as exhibiting mechanical flexibility for the kind of applications of interest in this section. In terms of implementation, diverse techniques can be identified including physical and solution mixing as well as in situ polymerization. ${ }^{59}$ Yet more efficient and promising techniques has been developed to increase manufacturability and performance including screen ${ }^{60,61}$ and inkjet ${ }^{62,63}$ printing techniques and even roll-to-roll manufacturing. ${ }^{64}$ In term of materials used to form composites, some well-known examples are polyaniline (PANI), polythiophene (PTH) and poly (3, 4-ethylenedioxythiophene):poly (styrenesulfonate) (PEDOT:PSS) as conductive polymers, and nanostructured Au, Ag, Bi, Te, $\mathrm{PbTe}, \mathrm{Sb}_{2} \mathrm{Te}_{3}, \mathrm{Bi}_{2} \mathrm{Te}_{3}$ and their alloys, as well as carbon nanotubes (CNT), as common inorganic TE materials. ${ }^{55,59,65}$ Current research in polymer-based and composite-based TEGs is focusing on finding novel strategies to further enhance their figure of merit (ZT), such including low-dimensional TE materials, as mentioned in the previous section, or including additional treatment for encapsulation in order to mitigate the impact of humidity on the electrical conductivity. ${ }^{59,66}$

In the coming subsections we will discuss some of the most practical and novel implementation of mechanically flexible and stretchable TEGs, as well as their challenges and the advantages and applications that these devices could enable.

Flexible thermoelectric generators for wearable and implantable biomedical applications.-The constantly increasing demand for low-cost and mobile personalized healthcare devices has driven re- searchers' efforts to produce wearable and implantable body sensor networks. However, new generations of wearable smart devices require high sampling rates resulting in high-energy consumption that limits the battery lifetime. In this sense, flexible thermoelectric generators can provide a method to increase the energy production capabilities of wearable and implantable electronics. For example, recently, Galo et al. have developed a method to integrate bismuth and antimony telluride TEGs on flexible silicon platforms. ${ }^{67}$ These TEGs show power densities in the order of $140 \mu \mathrm{W} \mathrm{cm}{ }^{-2}$. Here, the developed TEGs are based only on mature nanofabrication methods and materials, which allows high-performance TEGs to be integrated monolithically with silicon-based circuits. Additionally, due to the flexibility given by the thin silicon substrate, these devices can be used for wearable and implantable electronics where conformal integration of devices is required to take advantage of the waste heat generated by the human body. In 2011, Francioso et al. developed a flexible TEG based on bismuth and antimony telluride with a power output of $4.18 \mathrm{nW}$ at a temperature difference of $15^{\circ} \mathrm{C} .^{68}$ Later, Yang et al. showed a flexible thermoelectric nanogenerator (TENG) based on a Te-nanowire/poly(3-hexylthiophene) (P3HT) polymer composite, producing a current density of $32 \mathrm{nA} \mathrm{mm}^{-2}$ under a temperature difference of $55^{\circ} \mathrm{C}$, which can be also used as self-powered temperature sensor with a sensitivity of $0.15^{\circ} \mathrm{C}$ in ambient atmosphere. ${ }^{69}$ Rollto-roll processing has also been demonstrated for fast manufacturing through the screen printing of a polymeric TE material (PEDOT:PSS) and the printing of nanoparticle silver ink as electrodes. Although it demonstrates an efficient path to connect a large number of devices, the achieved performance is still very low (output power in the range of pW).$^{64}$ More recently, Madan et al. demonstrated a novel method to integrate printing methods with thermoelectric generators fabrication. Madan's devices can produce an output power in the range of $\mu \mathrm{W}$ at a temperature difference of $20^{\circ} \mathrm{C} .^{70}$ Several other demonstrations have shown flexible TEGs capable of producing higher power outputs (in the range of hundreds of $\mu \mathrm{W} \mathrm{cm}{ }^{-2}$ ) at relatively low-temperature differences. ${ }^{71,61,72}$ (Figure 2 shows one example) These devices have demonstrated the ability of simple and low-cost fabrication methods to create high-performance flexible TEGs that have advantages such as compact, silent, and high reliability. Also, in terms of power constraints, studies have suggested that a power source in the range of $100 \mu \mathrm{W} \mathrm{cm}{ }^{-2}$ at $1 \mathrm{~V}$ will be ideal for wearable sensor networks. ${ }^{73-75}$ However, while the heat of the human body is capable of providing a flexible TEG with power densities ranging from $100 \mu \mathrm{W} \mathrm{cm} \mathrm{cm}^{-2}$ to $1 \mathrm{~mW} \mathrm{~cm}{ }^{-2}$, such power outputs will cause a significant discomfort to the user due to a cold sensation on the skin. For these reasons, it is important to consider that TEGs are not likely to replace batteries in wearable devices, but instead, they can be used as a supplemental power source to extend the battery lifetime of state-of-the-art wearable sensor networks.

An additional interesting approach involve the use of paper as an affordable substrate alternative. Paper is a wide-spread and inexpensive material, whose mechanical properties are of interest and, in fact, it has been the focus of several studies that demonstrate diverse applications ranging from electronics, displays, RFID tags and sensors to energy harvesting and storage devices. ${ }^{76}$ Its use in the development of TEGs is also of great interest, as it has been shown to help improving the TE properties of PEDOT:PSS by reducing its thermal conductivity, due to the fibers interfering with phonon transport, and also enhancing its Seebeck coefficient, since the mean carrier energy of the carriers flow is increased. ${ }^{77}$ Recently, Sun et al. demonstrated an interesting approach to fabricate paper-based TEGs by simply impregnating paper with $\mathrm{n}$ - or $\mathrm{p}$-type colloidal semiconductor quantum dots, exhibiting power factors of $1 \times 10^{-5} \mathrm{~W} \mathrm{~m}^{-1} \mathrm{~K}^{-2}$ for the p-type material and $0.5 \times 10^{-5} \mathrm{~W} \mathrm{~m}^{-1} \mathrm{~K}^{-2}$ for the n-type material. ${ }^{78}$ Likewise, even high power generation has been demonstrated by screen printing a polymer-based TEG on a paper substrate, showing up to $50 \mu \mathrm{W}$ at a temperature difference of $100^{\circ} \mathrm{C}$, which was used to power up a light-emitting diode (LED) ${ }^{60}$ Alternatively, an inorganic-based TEG has been also demonstrated on a paper substrate by using a simple approach to achieve a power of $80 \mathrm{nW}$ at a temperature difference 


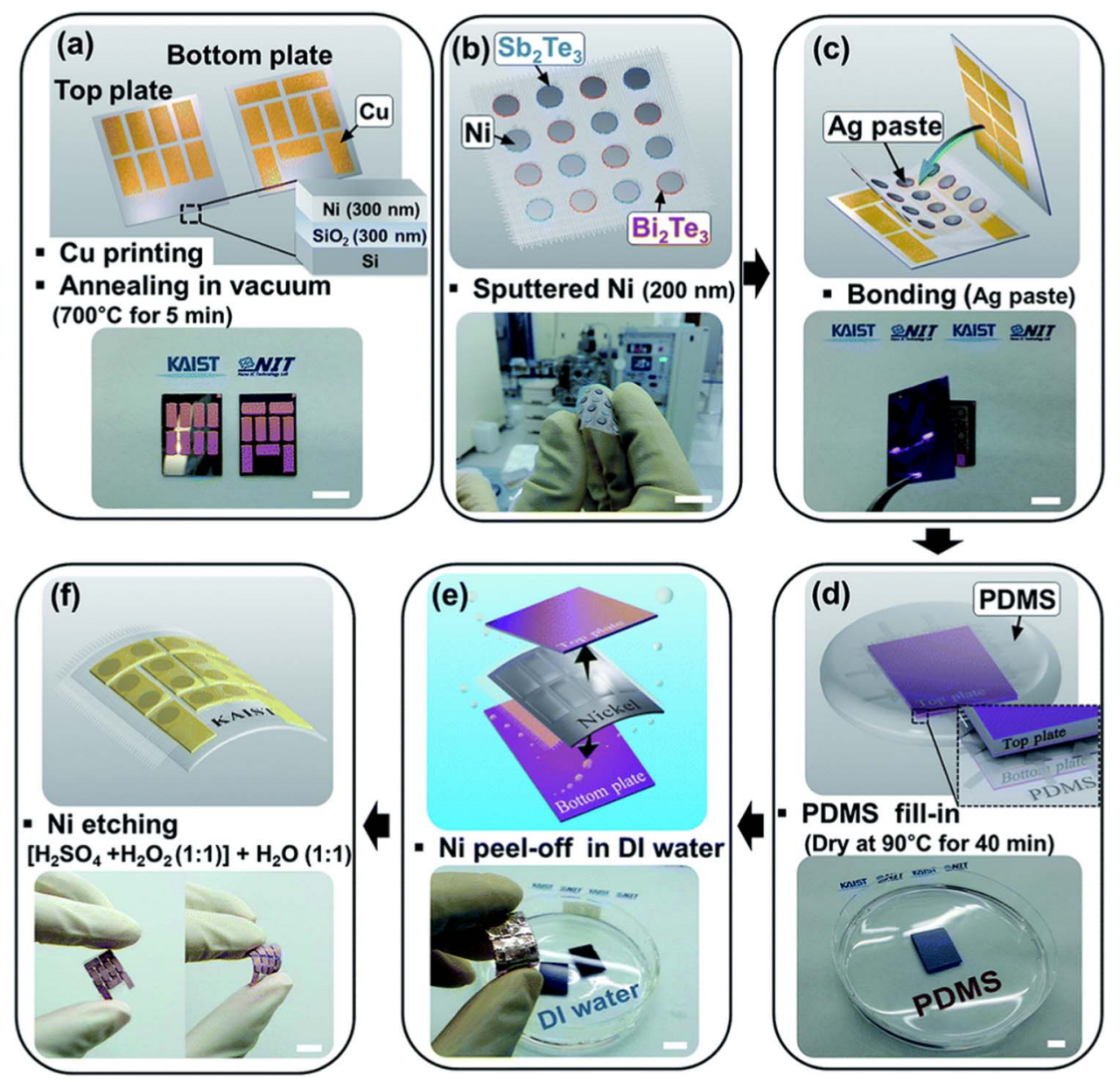

Figure 2. Common fabrication process of flexible thermoelectric generators; (a) preparation of Cu electrodes on a $\mathrm{Ni} / \mathrm{SiO}_{2} / \mathrm{Si}$ substrate. (b) Deposition of a Ni layer on $\mathrm{Bi}_{2} \mathrm{Te}_{3}$ and $\mathrm{Sb}_{2} \mathrm{Te}_{3}$ thick films to reduce contact resistance. (c) Bonding process of top plate/TE film/bottom plate using Ag paste. (d) Infiltrating the elastic polymer (PDMS) into the gap between the top and bottom plates. (e) Removal of the top and bottom plates by Ni peel-off in DI water. (f) Removing the remaining Ni layers by etching in a diluted mixture of sulfuric acid and hydrogen peroxide. Scale bar, $1 \mathrm{~cm}$. Reproduced with permission from Ref. 61 .

of $75^{\circ} \mathrm{C}$ with only $20 \mathrm{TE}$ pairs. ${ }^{79}$ An important advantage of the use of paper is the possibility of employing out-of-the-box techniques, such origami and kirigami, to stack and fold the devices in an area-efficient way and thus improve their power density.

In the case of implantable thermoelectric generators, few reports have already shown the ability of these harvesters to improve the battery lifetime of implantable electronics. ${ }^{80,81}$ However, these implantable devices are rigid and do not allow a conformal integration with naturally occurring asymmetric surfaces of the human organs. Also, due to the low-temperature difference ${ }^{82}$ (maximum $8^{\circ} \mathrm{C}$ ) between the inner organs and the surface of the skin, TEGs require an optimized design and a high number of thermopiles to achieve enough energy production. In this sense, flexible substrates can be taken advantage of to produce a roll-based device with a high-density array of thermopiles that could allow output powers in the range of hundreds of $\mu \mathrm{W}$. Finally, although flexible thermoelectric generators set a step forward for future wearable and implantable electronics power generation, it is to be noted that flexible matching circuits and voltage regulators are also required to take complete advantage of the waste heat produced by the human body. For this reason, we expect that in future, flexible wearable and implantable electronics will need to be integrated monolithically or heterogeneously on flexible substrates and should include processing and power management circuits, and high-performance energy harvesters that can help increase the lifetimes of batteries.
Beyond flexibility: stretchable thermoelectric generators.-In an effort to increase the functionality, adaptability, and practicality of many electronic devices, including TEGs, there has been several attempts to go beyond mechanical flexibility and develop novel stretchable architectures. ${ }^{12}$ Many everyday situations and applications might require the close integration of electronics with complex, curvilinear and mobile systems, such the human body, or with softer, mechanically advantageous materials such polymers, elastomers, rubbers, etc. However, the rigid nature of current conventional technologies poses a great challenge to achieve a fully flexible and stretchable system. Some successful previous attempts of stretchable electronic demonstrations include the introduction of novel, naturally stretchable materials such polymers/organics, composites, 2-dimensional (2D) and 1-dimensional (1D) materials. ${ }^{11,83}$ On the other hand, innovative ways to engineer the mechanical structure of devices can be used with out-of-the-box geometries, such serpentine, horseshoe, spiral, helical, fractals and others ${ }^{84-87}$ (Some are depicted in Figure $3 \mathrm{a}-3 \mathrm{~d})$. These materials and strategies have been used to demonstrate stretchable systems for diverse applications, such wearable systems, robotics, bio-integrated electronics and many others. ${ }^{88-91}$

As we have discussed, TEGs might play an important role in wearable technologies since they can convert the heat generated by the body into electrical energy useful to power up sensors, logic circuits and communication systems. ${ }^{92}$ Acquiring the capability of stretching TEGs will enable an improved integration with the complex motions of the human body. Smart clothing, fabrics and textiles, which can be flexed and stretched to some extent, might be used for this pur- 
(a) Serpentine

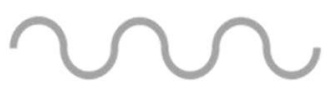

(c) Helix
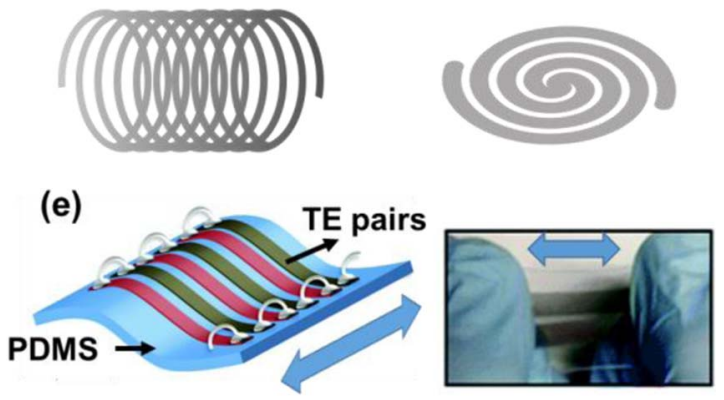

Figure 3. Schematics of some shapes used to implement stretchable electronics: (a) Serpentine, (b) horseshow, (c) helix and (d) spiral. (e) Stretchable TEG with TMDC nanosheets (TE pairs) on polymeric substrate (PDMS). Reproduced with permission from Ref. 99.

pose. In 2014 Kim et al. implemented a flexible and stretchy TEG by integrating a polymer-based fabric with inorganic TE materials to produce $224 \mathrm{nW}$ from the human body in an ambient temperature of $5^{\circ} \mathrm{C} .{ }^{93}$ More recently, Du et al. developed a power generating clothing by coating a commercial fabric with a TE polymer, that generated $12.5 \mathrm{nW}$ at a temperature difference of $75^{\circ} \mathrm{C} .{ }^{94}$ Other TEG devices have been demonstrated based on organic/polymeric TE materials with the potential to evolve into advanced stretchable devices, although their performance can be still greatly improved compared to inorganic TE materials. ${ }^{95,96}$ Another set of materials with potential stretchable capabilities are nano-engineered and 2D materials, which have shown encouraging predictions in terms of performance and power generation. That is the case of silicine ${ }^{97}$ and phosphorene, ${ }^{98}$ which can theoretically reach very high figures of merit (ZT), well above the unity. In terms of practical demonstrations, only very recently, Oh et al. developed a stretchable and foldable TEG based on transition metal dichalcogenide (TMDC) nanosheets (Figure 3e). Although functionality was demonstrated even at mechanical deformation of the device, with a maximum generated power of $38 \mathrm{nW}$ at a temperature difference of $60^{\circ} \mathrm{C}$ while at rest, they observed a very strong power degradation while stretching. ${ }^{99}$

Alternatively, and in a similar fashion as performed by Kim et al., ${ }^{93}$ we can rely on the higher TE properties of inorganic materials for power generation, while relying on organic/polymeric materials for mechanical support, and for flexing and stretching capabilities. Furthermore, novel shapes such as the ones described before (Figure 3a-3d) could potentially be used to develop stretchable TEGs and, at the same time, they might help to reduce mechanical stress on the inorganic materials and thus reduce the impact on the TE properties while deforming/stretching. ${ }^{84}$

An additional advantage of developing a stretchable TEG is the ability to adjust its separation between the cold and hot junction. Naturally, the further away you move from a heat source, the higher would be the temperature difference, which can lead to higher power generation as long as the TE properties are not highly impacted by the device's deformation. Recently, a spiral-based architecture was used to demonstrate this effect on a TEG made from $\mathrm{Bi}_{2} \mathrm{Te}_{3} / \mathrm{Sb}_{2} \mathrm{Te}_{3}$ sputtered on top of polyimide (PI) and polyester-paper. The maximum generated power increased by more than twice at $60 \%$ of elongation on the TEG fabricated on polyester-paper, a material with a lower thermal conductivity to ensure that the higher temperature difference is maintained. ${ }^{100}$ In fact, this characteristic could be really useful in many practical scenarios, such as in moving engines, robotic parts, and others where a heat source and heat sink might be available in the system but their separation could be long and in constant movement. In such cases, a stretchable TEG could be used to leverage the situation and produce the highest possible power.

\section{Additional Promising TEG-based Technologies}

Nano-manufactured glass windows for solar-based mass scale thermoelectric generation.-The promise of an un-interrupted supply of energy from photovoltaic solar panels is severely impeded by the intermittent nature of available sunlight. Inayat et al. ${ }^{56,101}$ have implemented novel thermoelectric glass windows for mass scale energy generation using the temperature difference between the rich supplies of solar heat and the ubiquitous ambient environment around us. These novel energy generators scavenge electricity from direct solar irradiance during daytime, while during off sun hours are still capable of utilizing the hot outdoor temperature to generate ample amount of electricity.

Thermoelectric systems are either fabricated to produce milli-watts of energy using a small temperature gradient of only a few Kelvins to power micro devices. On the other hand, thermoelectric generators in industrial applications or engines use temperature gradients of hundreds of Kelvins to produce kilowatts of energy. These thermoelectric windows extend the concept of TE systems at lower end of the spectrum (used for micro-devices), to a large coverage area, using abundant and unlimited source of temperature gradient between outside solar and ambient inside, for mass scale energy generation.

Expanding the concept of micro TEGs onto large coverage areas simultaneously eliminate the two primary problems associated with the micro TEGs. First, the low temperature gradient produces a very small output voltage in a micro TEG. This low output requires voltage boosters that are not only miniaturized but should also be highly efficient. In addition, designing and integrating power conditioning circuitry at micro scale becomes a hard challenge. By expanding thermoelectric system over a large coverage multiplies the output voltage manifolds, thus eliminating the need for voltage booster altogether or at least relaxing the efficiency specs for these boosters. Additionally, the macro sized mass scale energy harvesters also allow the use of large sized maximum power point trackers (MPPT) with high efficiency.

Design limitation of commonly available TEGs have served as a major impediment toward the employment of thermoelectric systems for mass scale energy generation utilizing the temperature gradients existing between outdoor (environment) and indoor (inside room). ${ }^{56,101}$ Lateral thermoelectric generators demonstrated by $\mathrm{H}$. Glosch $^{102}$ and D.M. Rowe ${ }^{103}$ require the heat to flow through the thermocouples in lateral direction. Alternatively, the vertical TEGs with vertically erected thermocouples similar to the devices implemented by H. Bottner for Micropelt, ${ }^{104}$ G.J. Snyder for JPL, ${ }^{105}$ M. Kishi for Seiko, ${ }^{106}$ require the flow of heat from top to bottom or vice versa through the device. Both these design versions strictly require the two counter temperature environments to be simultaneously accessible to the hot and cold side of the generator, thus, eliminating the presence of any interface between the two temperature environments. Given these conventional design limitations, interfaces like window glasses tend to make it impossible to generate thermoelectricity using the rich supply of hot outdoor environment and indoor ambient. By placing the thermoelectric generator on the outer side of a window (or vice versa) allows the outdoor temperature to influence the hot side of the generator but prevents the thermoelectric generator from accessing the cold indoor because of the intermediate blocking window. Inayat et al. ${ }^{56,101}$ have, however, been able to generate mass scale thermoelectricity by innovatively using the interface, between outdoor solar heat and indoor ambient, as generator of thermoelectricity by placing the thermoelectric materials through the interface.

The validity of the concept was verified with the finite element behavioral analysis of a thermoelectric window system. The model was designed for a single pair of complimentary thermoelectric legs (pillars) integrated inside a glass substrate. The two pillar device 

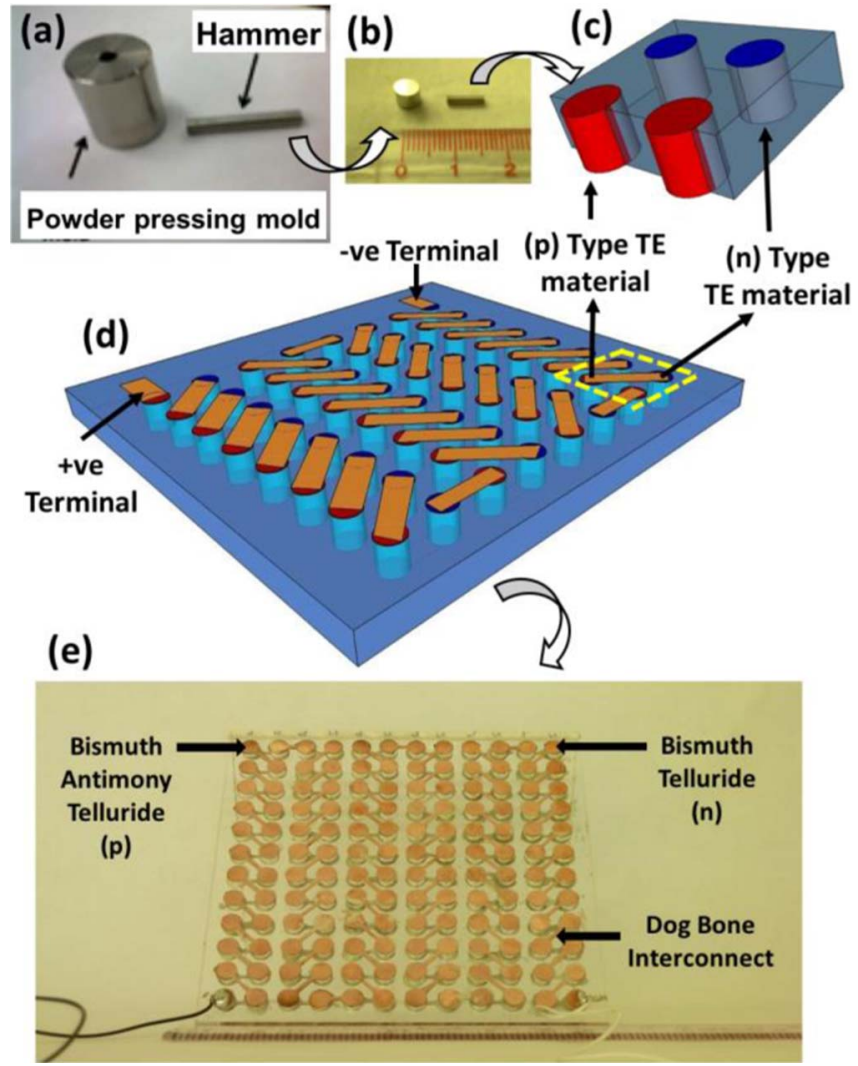

Figure 4. a) Stainless steel mold assembly for hot pressing of thermoelectric powders. Reproduced with permission from Ref. 56, b) hot pressed thermoelectric pillars, c) blow up schematic of window glass embedded with thermoelectric pillars, d) schematic of electrically interconnected thermoelectric pillars array integrated into window glass for mass scale energy generation, and, e) Plexiglas based proto-type thermoelectric window.

model used can be further extrapolated to give the outcome for a full sized glass window with thermoelectric materials integrated across the entire coverage of the glass window. The presence of an output voltage of $0.12 \mathrm{mV}$ on the $\mathrm{p}$ type pillar while $-0.04 \mathrm{mV}$ on the $\mathrm{n}$ type pillar of the device for a $20^{\circ} \mathrm{C}$ temperature gradient, confirms the validity of the model for predicting the behavior of a thermoelectric system, with thermoelectric materials embedded inside a glass window. ${ }^{107}$

Based on trade-offs between thermoelectric properties and the processing delay involved in mechanical alloying of large amounts of powders, as purchased powders were selected for batch nanomanufacturing of thermoelectric pillars (Figure 4). The as purchased thermoelectric powders were hot pressed into vertical pellets. The tubular design of the dies facilitates the thermoelectric characterization of the pressed pillars by eliminating the unnecessary delay involved in cutting smaller pellets out of a large tablet obtained using conventional large sized dies. Prototype implementation of a thermoelectric window has been implemented with $\left(132.25 \mathrm{~cm}^{2}\right)$ Plexiglas panel laser drilled with 144 holes to accommodate hot pressed thermoelectric pillars.

The output power from the large Plexiglas panel integrated with hot pressed pillars of as-purchased powders of the two materials was $0.16 \mu \mathrm{W}$ for a temperature difference of $22.5^{\circ} \mathrm{C}$ between the hot and the cold side (Figure 5). ${ }^{101}$

Next, Inayat et al., ${ }^{56,101}$ leveraged the mechanical alloying of commercially available powders of $\mathrm{Bi}_{1.75} \mathrm{Te}_{3.25}$ and $\mathrm{Sb}_{2} \mathrm{Te}_{3}$ to nanomanufacture pellets through ball milling for high performance thermoelectric windows. Effect of mechanical alloying and hot pressing on thermoelectric properties of the as-purchased powders was investigated through the thermoelectric characterization of the hot pressed pillars of the as purchased powder samples. Nano-manufacturing

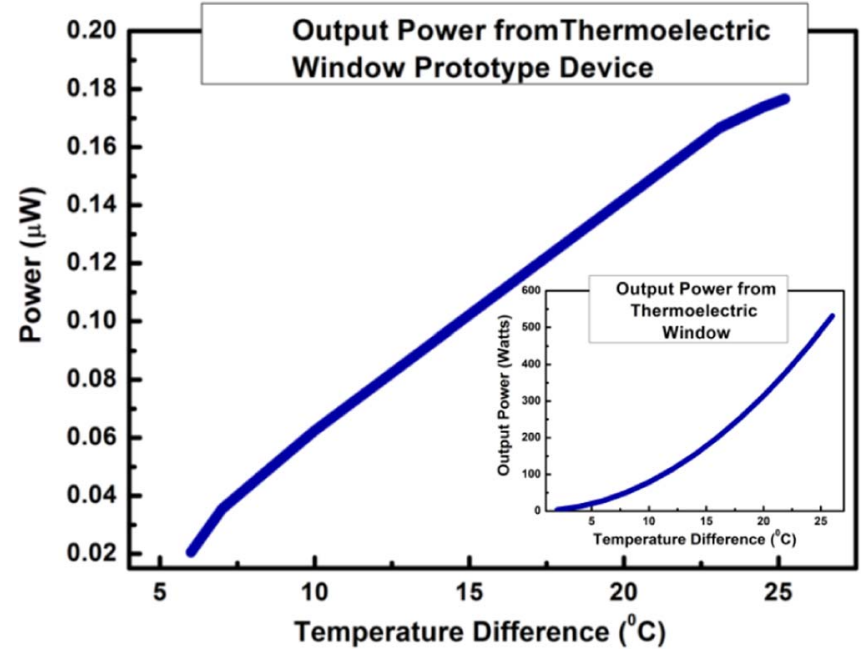

Figure 5. Output power from a Plexiglas proto-type thermoelectric window. Reproduced with permission from Ref. 101. Inset shows projected output power levels for a $9 \mathrm{~m}^{2}$ thermoelectric window.

through mechanical alloying showed a significant enhancement in electrical and thermal properties of the as purchased powder samples.

Although the output power levels from the prototype panel were low, the presence of highly repeatable thermoelectric output from the prototype thermoelectric Plexiglas validated the effectiveness of a batch fabrication process enabled efficient nano-manufacturing of large batches of thermoelectric pillars for large coverage thermoelectric window. Additionally, the property enhancement of thermoelectric powders with mechanical alloying also opened up an opportunity for further investigation into the ball milling of thermoelectric powders to bring a significant increase in the output power levels.

Maximum attainable limit of the hot press used and the contact resistance between the thermoelectric pillars and the metal interconnects have been the major performance impeding factors for the prototype thermoelectric panel. By using techniques like Spark Plasma Sintering (SPS) for performance enhancement of thermoelectric pillars and inkjet printing for low resistance interconnects, the work of Inayat et al. offers an opportunity to potentially generate $304 \mathrm{~W}$ of usable power from $9 \mathrm{~m}^{2}$ window at a $20^{\circ} \mathrm{C}$ temperature gradient. ${ }^{56}$ If a natural temperature gradient exists, this can serve as a sustainable energy source for green building technology.

Thermoelectricity for regenerative computing.- It is now a wellknown fact that the classical 'Moore's Law' gate length scaling of silicon transistors has plateaued. Due to difficulty in advancing process technology as well as fundamental physics bottlenecks, the computing industry is now shifting away from a tick-tock process-architecture model to a process-architecture-optimization model. What this means is that future integrated-circuit technology nodes (down to $7 \mathrm{~nm}$ ) will be around for a longer time and integrated device manufacturers may determine their own roadmap based on the requirements of system integrators. The focus of major system integrators is largely in mobile and distributed computing, given the growing consumer demands for faster and efficient electronics in an attractive form-factor. That being said, historically, there has always been a trade-off with power consumption and performance in mobile electronics, where usually the form-factor is the determining factor. At the higher levels, optimized circuit blocks and software plays a huge role in narrowing this performance-power gap. However, to fundamentally affect change and achieve leap-bound performance-power gains, new innovation is required at the transistor level. The paradigm-shift from a planar topology to a three-dimensional architecture (FinFETs), is an excellent example. Compared to past planar transistors, current generation of FinFETs ${ }^{108}$ are faster while consuming less power and 

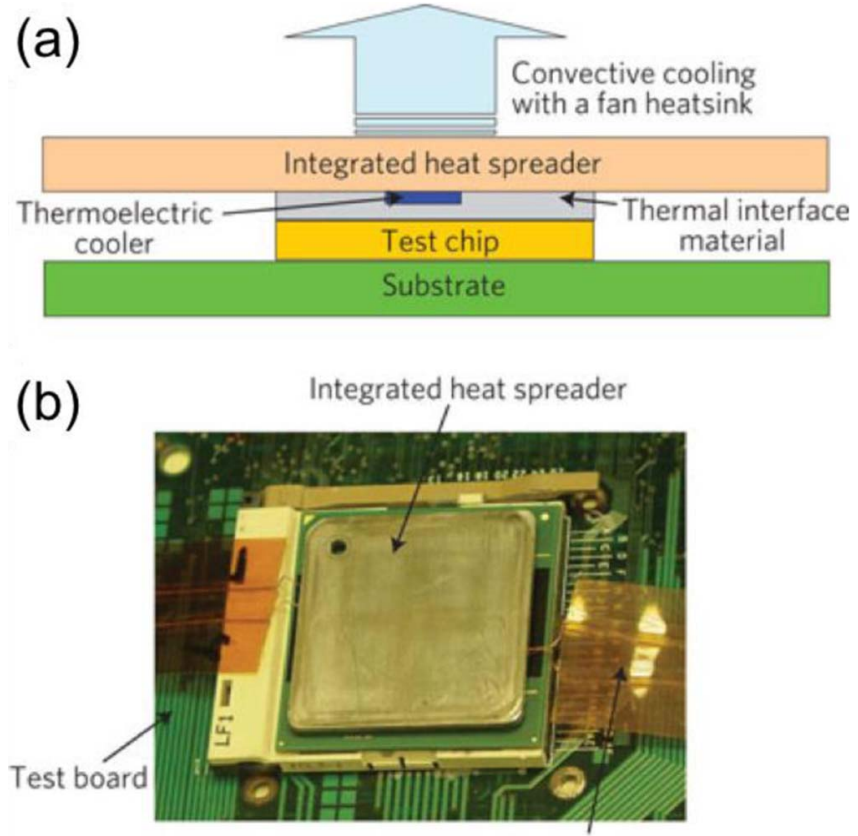

Electrical connection to thermoelectric cooler

Figure 6. (a) Cross-section schematic of thermoelectric cooler integrated with test chip. (b) Digital photograph of the setup shown in (a) with the thermoelectric cooler beneath the heat spreader. Reproduced with permission from Ref. 112.

also run cooler temperatures due to lower heat dissipation. But due to the rising cost and complexity in manufacturing FinFETs on bulk silicon, there is a wide consensus in migrating to silicon on insulator (SOI) platforms for future technology nodes. Heat dissipation and transport in SOI based nanoscale transistors is more challenging due to the presence of an insulating buried silicon dioxide layer. ${ }^{109,110}$ This is even more profound in stacked 3-D integrated circuits ${ }^{111}$ that are being considered for unprecedented computing parallelism and speed. The bottom line is that heat dissipation from such nanoscale devices will persist as transistor density continues to increase. The way this heat flux is managed in electronics will ultimately determine their performance, power consumption, operation life-time and reliability.

One way to address the problems due to heat dissipation is by effectively removing this heat flux using thermoelectric coolers. Chowdhury et al. demonstrated chip level site-specific cooling by making use of nanostructured thin-film super-lattices of $\mathrm{Bi}_{2} \mathrm{Te}_{3}$ to remove dissipated heat from a silicon test chip containing micro-heaters (Figure 6). ${ }^{112}$ Using this, they were able to demonstrate nearly $15^{\circ} \mathrm{C}$ cooling on the test chip with a heat flux of $\sim 1300 \mathrm{~W} \mathrm{~cm}^{-2}$. Another way to aid tackling the heat dissipation problem as well as boost battery lifetime in mobile electronics, is by harvesting and reusing this heat at the device, circuit or die level. Using commercial off-the-shelf thermoelectric generators (TEG), Zhou et al. carried out experiments to measure and harvest the heat energy dissipated by microprocessors at different workloads showing the un-tapped potential of thermoelectricity in computing. ${ }^{113}$ Building on this concept, in another work, Fahad et al. introduced the concept of quasi self-powered integrated circuits by reusing the harvested waste heat (Figure 7a-7b) from microprocessors, ${ }^{114}$ to drive an array of light emitting diodes (LED). However, these are all examples of off-chip/die implementations of standalone thermoelectric modules. A more fundamental and impactful approach would be at the device and circuit level, by integration of thermoelectric elements alongside transistor blocks as well as metal interconnects. A great example of this is demonstrated by Aktakka et al., where low temperature high quality $\mathrm{Bi}_{2} \mathrm{Te}_{3}$ and $\mathrm{Sb}_{2} \mathrm{Te}_{3}$ thin film (a)

(b)

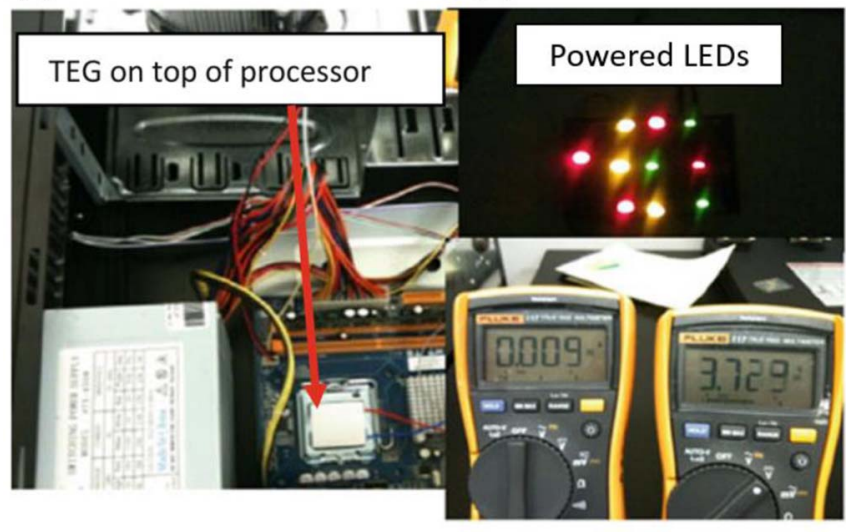

(c) Planar Thermoelectric Harvester CMOS FinFET
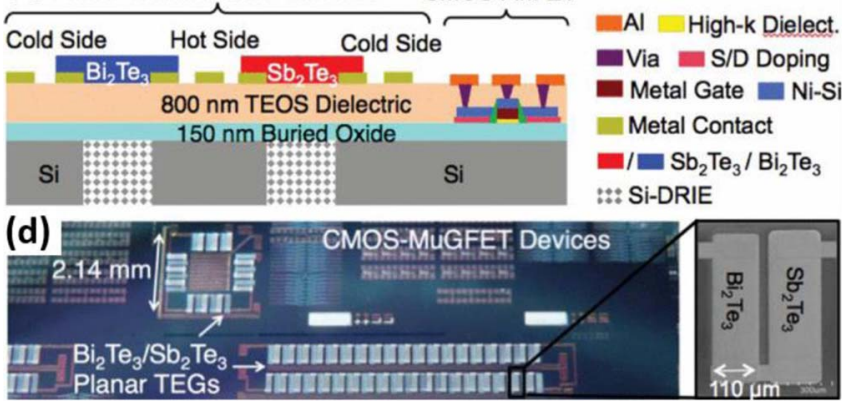

Figure 7. (a) Experimental setup of TEG mounted on top of processor. (b) Digital photograph of powered LEDs and multimeters showing current and voltage generation. ${ }^{114}$ (d) Cross-section schematic of integrated TEG device with CMOS FinFET. (d) Digital photograph of TEGs co-integrated with CMOS FinFET. Reproduced with permission from Ref. 115.

thermoelectric devices were integrated with SOI FinFETs (Figure 7c7d) to harvest $\sim 0.7 \mu \mathrm{W}$ of power from a $\sim 21^{\circ} \mathrm{C}$ thermal gradient. ${ }^{115}$ Similar innovations can enable the development of quasi self-powered electronics for the ever growing mobile consumer market.

\section{Future Outlook and Conclusions}

As we began this review, we suggested the need for seamless ultra-light weight micro to milli-scale power supplies for emerging electronic applications. Efficient energy harvesters with ultra-large capacity (as well as ultra-light weight) energy storage can be significantly useful to meet that demand. However, there is still a long way to go to achieve such goal. In that regard, thermoelectric generators have been identified as an alternative energy source with great potential. However, its efficiency needs to be improved significantly. In that pursuit, constant development of nanotechnology and nanomaterials has been greatly benefiting TEG technology. Furthermore, the engineering challenges that are coming with emerging technologies, such as wearable electronics, bio-integrated systems, robotics, cybernetics and others, lead the way to novel engineering approaches and innovative devices that are the focus of this review, such the introduction of mechanically reconfigurable and adaptable devices. Looking forward to the continuing development of thermoelectric, some applications have been discussed, such as the integration of TE materials in windows for mass-scale electricity production and in current- and next-generation computing for efficient cooling and energy harvesting.

\section{References}

1. A. K. Akella, R. P. Saini, and M. P. Sharma, Renew. Energy, 34, 390 (2009).

2. X. F. Zheng, C. X. Liu, Y. Y. Yan, and Q. Wang, Renew. Sustain. Energy Rev., 32, 486 (2014) 
3. D. M. Rowe, Renew, Energy, 16, 1251 (1999).

4. B. I. Ismail and W. H. Ahmed, Recent Patents Electr. Eng., 2, 27 (2009).

5. S. B. Riffat and X. Ma, Appl. Therm. Eng., 23, 913 (2003).

6. A. Bulusu and D. G. Walker, Superlattices Microstruct., 44, 1 (2008).

7. M. H. Elsheikh, D. A. Shnawah, M. F. M. Sabri, S. B. M. Said, M. H. Hassan, M. B. A. Bashir, and M. Mohamad, Renew. Sustain. Energy Rev., 30, 337 (2014).

8. C. A. Gould, N. Y. A. Shammas, S. Grainger, and I. Taylor, in 26th Int. Conf. Microelectron. MIEL., p. 329, Nis, Serbia (2008).

9. G. J. Snyder and E. S. Toberer, Nat. Mater, 7, 105 (2008).

10. H. Alam and S. Ramakrishna, Nano Energy, 2, 190 (2013).

11. S. Choi, H. Lee, R. Ghaffari, T. Hyeon, and D.-H. Kim, Adv. Mater, 28, 4203 (2016)

12. J. A. Rogers, T. Someya, and Y. Huang, Science, 327, 1603 (2010).

13. J. B.-H. Tok and Z. Bao, Sci. China Chem., 55, 718 (2012).

14. J. R. Sootsman, D. Y. Chung, and M. G. Kanatzidis, Angew. Chemie Int. Ed., 48 8616 (2009)

15. D. M. Rowe (ed.), Handbook of Thermoelectrics: Macro to Nano, CRC Press, Boca Raton, (2005).

16. A. F. Ioffe, Semiconductor thermoelements, and Thermoelectric cooling, Infosearch, ltd., London, (1957).

17. F. DiSalvo, Science, 285, 703 (1999).

18. J. Yang, J. Electron. Mater, 36, 703 (2007)

19. J. Yang and T. Caillat, MRS Bull., 31, 224 (2006)

20. D. M. Rowe (ed.), Handbook of Thermoelectrics, CRC Press, Boca Raton, (1995).

21. K. Yazawa and A. Shakouri, in Proc. ASME Int. Mech. Eng. Congr. Expo., p. 569 Vancouver, Canada (2010).

22. A. Majumdar, Science, 303, 777 (2004).

23. J. P. Heremans, M. S. Dresselhaus, L. E. Bell, and D. T. Morelli, Nat. Nanotechnol. 8, $471(2013)$

24. Y. Pan and J.-F. Li, NPG Asia Mater., 8, e275 (2016).

25. C. J. Vineis, A. Shakouri, A. Majumdar, and M. G. Kanatzidis, Adv. Mater, 22, 3970 (2010)

26. C. W. Li, J. Hong, A. F. May, D. Bansal, S. Chi, T. Hong, G. Ehlers, and O. Delaire Nat. Phys., 11, 1 (2015)

27. A. I. Boukai, Y. Bunimovich, J. Tahir-Kheli, J.-K. Yu, W. A. Goddard, and J. R. Heath, Nature, 451, 168 (2008).

28. S. H. Lee, S. Y. Jang, J. W. Roh, J. Park, and W. Lee, INEC 2010 - 2010 3rd Int. Nanoelectron. Conf. Proc., 1203 (2010).

29. E. B. Ramayya, L. N. Maurer, A. H. Davoody, and I. Knezevic, Phys. Rev. B, 86 115328 (2012).

30. X. Zhang and L.-D. Zhao, J. Mater., 1, 92 (2015).

31. E. Z. Xu, Z. Li, J. A. Martinez, N. Sinitsyn, H. Htoon, N. Li, B. Swartzentruber, J. A. Hollingsworth, J. Wang, and S. X. Zhang, Nanoscale, 7, 2869 (2015).

32. H. Yang, J. H. Bahk, T. Day, A. M. S. Mohammed, G. J. Snyder, A. Shakouri, and Y. Wu, Nano Lett., 15, 1349 (2015).

33. R. Venkatasubramanian, E. Siivola, T. Colpitts, and B. O'Quinn, Nature, 413, 597 (2001).

34. T. C. Harman, P. J. Taylor, D. L. Spears, and M. P. Walsh, J. Electron. Mater, 29, L1 (2000).

35. J.-K. Yu, S. Mitrovic, D. Tham, J. Varghese, and J. R. Heath, Nat. Nanotechnol., 5, 718 (2010).

36. H. Sadeghi, S. Sangtarash, and C. J. Lambert, Beilstein J. Nanotechnol., 6, 1176 (2015).

37. S. Yamasaka, K. Watanabe, S. Sakane, S. Takeuchi, A. Sakai, K. Sawano, and Y. Nakamura, Sci. Rep., 6, 22838 (2016).

38. Y. Pei, H. Wang, and G. J. Snyder, Adv. Mater, 24, 6125 (2012).

39. Q. Zhang, H. Wang, W. Liu, H. Wang, B. Yu, Q. Zhang, Z. Tian, G. Ni, S. Lee, K. Esfarjani, G. Chen, and Z. Ren, Energy Environ. Sci., 5, 5246 (2012).

40. Q. Zhang, B. Liao, Y. Lan, K. Lukas, W. Liu, K. Esfarjani, C. Opeil, D. Broido, G. Chen, and Z. Ren, Proc. Natl. Acad. Sci., 110, 13261 (2013).

41. L. D. Zhao, H. J. Wu, S. Q. Hao, C. I. Wu, X. Y. Zhou, K. Biswas, J. Q. He, T. P. Hogan, C. Uher, C. Wolverton, V. P. Dravid, and M. G. Kanatzidis, Energy Environ. Sci., 6, 3346 (2013).

42. A. J. Minnich, M. S. Dresselhaus, Z. F. Ren, and G. Chen, Energy Environ. Sci., 2, 466 (2009).

43. D. O. Demchenko, P. D. Heinz, and B. Lee, Nanoscale Res. Lett., 6, 502 (2011)

44. A. I. Hochbaum, R. Chen, R. D. Delgado, W. Liang, E. C. Garnett, M. Najarian, A. Majumdar, and P. Yang, Nature, 451, 163 (2008).

45. K. Zhang, J. Qiu, and S. Wang, Nanoscale, 8, 8033 (2016)

46. N. Toshima, K. Oshima, H. Anno, T. Nishinaka, S. Ichikawa, A. Iwata, and Y. Shiraishi, Adv. Mater, 27, 2246 (2015).

47. C. Cho, B. Stevens, J. H. Hsu, R. Bureau, D. A. Hagen, O. Regev, C. Yu, and J. C. Grunlan, Adv. Mater., 27, 2996 (2015).

48. K. Zhang, M. Davis, J. Qiu, L. Hope-Weeks, and S. Wang, Nanotechnology, 23 385701 (2012)

49. C. Meng, C. Liu, and S. Fan, Adv. Mater, 22, 535 (2010).

50. Z. H. Ge, L. D. Zhao, D. Wu, X. Liu, B. P. Zhang, J. F. Li, and J. He, Mater. Today, 19, 227 (2016).

51. O. Bubnova and X. Crispin, Energy Environ. Sci., 5, 9345 (2012)

52. W. Zeng, L. Shu, Q. Li, S. Chen, F. Wang, and X. Tao, Adv. Mater., 26, 5310 (2014).

53. Y. Chen, Y. Zhao, and Z. Liang, Energy Environ. Sci., 8, 401 (2015).

54. M. He, F. Qiu, and Z. Lin, Energy Environ. Sci., 6, 1352 (2013).

55. J.-H. Bahk, H. Fang, K. Yazawa, and A. Shakouri, J. Mater. Chem. C, 3, 10362 (2015).

56. S. B. Inayat, K. R. Rader, and M. M. Hussain, Sci. Rep., 2, 841 (2012).

57. W. Qu, M. Plötner, and W.-J. Fischer, J. Micromech. Microeng., 11, 146 (2001).
58. O. Bubnova, Z. U. Khan, A. Malti, S. Braun, M. Fahlman, M. Berggren, and X. Crispin, Nat. Mater, 10, 429 (2011).

59. Y. Du, S. Z. Shen, K. Cai, and P. S. Casey, Prog. Polym. Sci., 37, 820 (2012).

60. Q. Wei, M. Mukaida, K. Kirihara, Y. Naitoh, and T. Ishida, RSC Adv., 4, 28802 (2014)

61. S. J. Kim, J. H. We, and B. J. Cho, Energy Environ. Sci., 7, 1959 (2014).

62. E. Drahi, A. Gupta, S. Blayac, S. Saunier, and P. Benaben, Phys. status solidi, 211, 1301 (2014).

63. Z. Lu, M. Layani, X. Zhao, L. P. Tan, T. Sun, S. Fan, Q. Yan, S. Magdassi, and H. H. Hng, Small, 10, 3551 (2014)

64. R. R. Søndergaard, M. Hösel, N. Espinosa, M. Jørgensen, and F. C. Krebs, Energy Sci. Eng., 1, 81 (2013).

65. B. T. McGrail, A. Sehirlioglu, and E. Pentzer, Angew. Chem. Int. Ed., 54, 1710 (2015)

66. M. A. Kamarudin, S. R. Sahamir, R. S. Datta, B. D. Long, M. F. Mohd Sabri, and S. Mohd Said, Sci. World J., 2013, 713640 (2013).

67. G. A. T. Sevilla, S. Bin Inayat, J. P. Rojas, A. M. Hussain, and M. M. Hussain, Small, 9, 3916 (2013)

68. L. Francioso, C. De Pascali, I. Farella, C. Martucci, P. Cretì, P. Siciliano, an A. Perrone, J. Power Sources, 196, 3239 (2011).

69. Y. Yang, Z.-H. Lin, T. Hou, F. Zhang, and Z. L. Wang, Nano Res., 5, 888 (2012).

70. D. Madan, Z. Wang, P. K. Wright, and J. W. Evans, Appl. Energy, 156, 587 (2015)

71. P. Sheng, Y. Sun, F. Jiao, C. Di, W. Xu, and D. Zhu, Synth. Met., 193, 1 (2014).

72. K. Kato, Y. Hatasako, M. Kashiwagi, H. Hagino, C. Adachi, and K. Miyazaki, $J$ Electron. Mater, 43, 1733 (2014).

73. C. Ho, M. Mark, M. Koplow, L. Miller, A. Chen, E. Reilly, J. Rabaey, J. Evans, and P. Wright, in 6th Int. Work. Wearable Implant. Body Sens. Networks, p. 29, Berkeley, CA (2009)

74. P. D. Mitcheson, in 32nd Annu. Int. Conf. IEEE Eng. Med. Biol., p. 3432, Bueno Aires, Argentina (2010)

75. T. Torfs, V. Leonov, C. Van Hoof, and B. Gyselinckx, in 5th IEEE Conf. Sensors, p. 427, Daegu, Korea (2006)

76. D. Tobjörk and R. Österbacka, Adv. Mater. 23, 1935 (2011).

77. Q. Jiang, C. Liu, J. Xu, B. Lu, H. Song, H. Shi, Y. Yao, and L. Zhang, J. Polym. Sci. Part B Polym. Phys., 52, 737 (2014).

78. C. Sun, A. H. Goharpey, A. Rai, T. Zhang, and D.-K. Ko, ACS Appl. Mater. Inter faces, $\mathbf{8}, 22182$ (2016).

79. J. P. Rojas, D. Conchouso, A. Arevalo, D. Singh, I. G. Foulds, and M. M. Hussain, Nano Energy, 31, 296 (2017).

80. Y. Yang, G. D. Xu, and J. Liu, J. Med. Device., 8, 14507 (2014).

81. Y. Yang, X.-J. Wei, and J. Liu, J. Phys. D. Appl. Phys., 40, 5790 (2007).

82. K. T. Settaluri, H. Lo, and R. J. Ram, J. Electron. Mater., 41, 984 (2012).

83. A. M. Hussain and M. M. Hussain, Adv. Mater, 28, 4219 (2016).

84. S. Wang, Y. Huang, and J. A. Rogers, IEEE Trans. Compon., Packag., Manuf. Technol., 5, 1201 (2015).

85. S. Xu, Z. Yan, K.-I. Jang, W. Huang, H. Fu, J. Kim, Z. Wei, M. Flavin, J. McCracken, R. Wang, A. Badea, Y. Liu, D. Xiao, G. Zhou, J. Lee, H. U. Chung, H. Cheng, W. Ren, A. Banks et al., Science, 347, 154 (2015).

86. J. P. Rojas, A. Arevalo, I. G. Foulds, and M. M. Hussain, Appl. Phys. Lett., 105, 154 (2014)

87. J. Fan, W.-H. Yeo, Y. Su, Y. Hattori, W. Lee, S.-Y. Jung, Y. Zhang, Z. Liu, H. Cheng, L. Falgout, M. Bajema, T. Coleman, D. Gregoire, R. J. Larsen, Y. Huang, and J. A. Rogers, Nat. Commun., 5, 3266 (2014).

88. J. Kim, J. Lee, D. Son, M. K. Choi, and D.-H. Kim, Nano Converg., 3, 1 (2016).

89. I. Jung, J. Xiao, V. Malyarchuk, C. Lu, M. Li, Z. Liu, J. Yoon, Y. Huang, and J. A. Rogers, Proc. Natl. Acad. Sci., 108, 1788 (2011).

90. D. Rus and M. T. Tolley, Nature, 521, 467 (2015).

91. L. Xu, S. R. Gutbrod, Y. Ma, A. Petrossians, Y. Liu, R. C. Webb, J. A. Fan, Z. Yang, R. Xu, J. J. Whalen, J. D. Weiland, Y. Huang, I. R. Efimov, and J. A. Rogers, $A d v$ Mater, 27, 1731 (2015)

92. V. Leonov and R. J. M. Vullers, J. Renew. Sustain. Energy, 1, 062701 (2009)

93. M.-K. Kim, M.-S. Kim, S. Lee, C. Kim, and Y.-J. Kim, Smart Mater. Struct., 23 105002 (2014).

94. Y. Du, K. Cai, S. Chen, H. Wang, S. Z. Shen, R. Donelson, and T. Lin, Sci. Rep., 5 $6411(2015)$

95. Q. Zhang, Y. Sun, W. Xu, and D. Zhu, Adv. Mater, 26, 6829 (2014).

96. P. Aranguren, A. Roch, L. Stepien, M. Abt, M. von Lukowicz, I. Dani, and D. Astrain, Appl. Therm. Eng., 102, 402 (2016)

97. H. Sadeghi, S. Sangtarash, and C. J. Lambert, Sci. Rep., 5, 9514 (2015).

98. J. Zhang, H. J. Liu, L. Cheng, J. Wei, J. H. Liang, D. D. Fan, J. Shi, X. F. Tang, and Q. J. Zhang, Sci. Rep., 4, 3621 (2014).

99. J. Y. Oh, J. H. Lee, S. W. Han, S. S. Chae, E. J. Bae, Y. H. Kang, W. J. Choi, S. Y. Cho, J -O. Lee, H. K. Baik, and T. Il Lee, Energy Environ. Sci.,9, 1696 (2016).

100. J. P. Rojas, D. Singh, D. Conchouso, A. Arevalo, I. G. Foulds, and M. M. Hussain, Nano Energy, 30, 691 (2016).

101. S. B. Inayat, K. R. Rader, and M. M. Hussain, Energy Technol., 2, 292 (2014)

102. H. Glosch, M. Ashauer, U. Pfeiffer, and W. Lang, Sensors Actuators A Phys., 74 246 (1999)

103. D. M. Rowe, D. V Morgan, and J. H. Kiely, Electron. Lett., 25, 166 (1989).

104. H. Bottner, J. Nurnus, A. Gavrikov, G. Kuhner, M. Jagle, C. Kunzel, D. Eberhard, G. Plescher, A. Schubert, and K. H. Schlereth, J. Microelectromechanical Syst., 13, 414 (2004).

105. G. J. Snyder, J. R. Lim, C.-K. Huang, and J.-P. Fleurial, Nat Mater, 2, 528 (2003).

106. M. Kishi, H. Nemoto, T. Hamao, M. Yamamoto, S. Sudou, M. Mandai, and S. Yamamoto, in 18th Int. Conf. Thermoelectr., p. 301, Baltimore, MD (1999). 
107. S. B. Inayat, Nano-Micro Materials Enabled Thermoelectricity From Window Glasses, thesis, King Abdullah University of Science and Technology (2012).

108. D. Hisamoto, W.-C. Lee, J. Kedzierski, H. Takeuchi, K. Asano, C. Kuo, E. Anderson, T.-J. King, J. Bokor, and C. Hu, IEEE Trans. Electron Devices, 47, 2320 (2000).

109. M. Asheghi, M. N. Touzelbaev, K. E. Goodson, Y. K. Leung, and S. S. Wong, J. Heat Transfer, 120, 30 (1998).

110. E. Pop, S. Sinha, and K. E. Goodson, Proc. IEEE, 94, 1587 (2006).

111. F. Tavakkoli, S. Ebrahimi, S. Wang, and K. Vafai, Int. J. Heat Mass Transf., 97, 337 (2016).
112. I. Chowdhury, R. Prasher, K. Lofgreen, G. Chrysler, S. Narasimhan, R. Mahajan, D. Koester, R. Alley, and R. Venkatasubramanian, Nat Nano, 4, 235 (2009)

113. Y. Zhou, S. Paul, and S. Bhunia, in Des. Autom. Test Eur. 2008, p. 98, Munich, Germany (2008).

114. H. Fahad, M. Hasan, G. Li, and M. Hussain, Appl. Nanosci., 3, 175 (2013)

115. E. E. Aktakka, N. Ghafouri, C. E. Smith, R. L. Peterson, M. M. Hussain, and K. Najafi, IEEE Electron Device Lett., 34, 1334 (2013). 